\title{
HOMOMORPHISMS AND IDEMPOTENTS OF GROUP ALGEBRAS
}

\author{
BY PAUL J. COHEN
}

Communicated by Walter Rudin, January 14, 1959

Let $G$ be a locally compact abelian group. We denote by $M(G)$ the algebra of all finite complex-valued Borel measures on $G$. The algebra is normed by assigning to each measure its total variation, and the product or convolution of the measures $\mu$ and $\nu$ is defined by

$$
(\mu * \nu)(E)=\iint_{x+y \in E} d \mu(x) d \nu(y) .
$$

If a particular Haar measure is chosen on $G$, the subalgebra of absolutely continuous measures may be identified with $L(G)$, the algebra of absolutely integrable functions. The Fourier transform of a measure $\mu$ is a function $\hat{\mu}$ defined on $\hat{G}$, the dual group of $G$, by the formula

$$
\hat{\mu}(\chi)=\int_{G}(\chi, g) d \mu(g),
$$

where $(\chi, g)$ denotes $\chi$ evaluated at $g$. Each $\chi$ thus yields a homomorphism of $M(G)$ onto the complex numbers. Every such homomorphism of $L(G)$ is obtained in this way.

Let $\phi$ be a homomorphism of $L(G)$ into $M(H)$. After composing with $\phi$, every homomorphism of $M(H)$ onto the complex numbers either is identically zero, or can be identified with a member of $\hat{G}$. We thus have a map $\phi_{*}$ from $\hat{H}$ into $\{\hat{G}, 0\}$, the union of $\hat{G}$ and the symbol 0 , the latter to be considered as the point at infinity. Our main result is:

THEOREM 1. For every homomorphism $\phi$ of $L(G)$ into $M(H)$, there exist a finite number of cosets of open subgroups of $\hat{H}$, which we denote by $K_{i}$, and continuous maps $\psi_{i}: K_{i} \rightarrow \hat{G}$, such that

$$
\psi_{i}(x+y-z)=\psi_{i}(x)+\psi_{i}(y)-\psi_{i}(z),
$$

with the following property: there is a decomposition of $\hat{H}$ into the disjoint union of sets $S_{j}$, each lying in the Boolean ring generated by the sets $K_{i}$, such that on each $S_{j}, \phi_{*}$ is either identically zero or agrees with some $\psi_{i}$, where $S_{j} \subset K_{i}$.

Conversely, for any such map of $\hat{H}$ into $\{\hat{G}, 0\}$, there is a homo- 
morphism of $L(G)$ into $M(H)$ which induces it. The map carries $L(G)$ into $L(H)$ if and only if $\phi_{*}^{-1}$ of every compact subset of $\hat{G}$ is compact.

The main tool in the proof of Theorem 1 is the following lemma:

LEMmA. If $G$ and $H$ are compact, then the graph of $\phi_{*}$, namely all pairs $\left(\phi_{*}(h), h\right)$ where $\phi_{*}(h)$ is not zero, is such that its characteristic function is the Fourier transform of a measure on $G \times H$.

The measure in the lemma must of course be an idempotent, that is, satisfy the equation $\mu * \mu=\mu$. The essential difficulty rests in the determination of all idempotent measures on a group.

Theorem 2. If $\mu$ is an idempotent measure, then $\hat{\mu}$ is the characteristic function of a subset $E$ of $\hat{G}$ which lies in the Boolean ring generated by cosets of open subgroups of $\hat{G}$.

It is not difficult to deduce Theorem 1 from the above statements in the case in which $G$ and $H$ are compact. In the general case one shows that there is a natural extension of $\phi$ to a homomorphism of $L(\bar{G})$ into $M(\bar{H})$ where $\bar{G}$ and $\bar{H}$ are the Bohr compactifications of $G$ and $H$ respectively. It can then be shown that if $\hat{G}$ and $\hat{H}$ are taken in the discrete topology, Theorem 1 holds. However we know that $\phi_{*}$ is continuous and after some manipulation we can show that Theorem 1 holds in the original form.

Both Theorems 1 and 2 were known in special cases before. We note that Theorem 2 implies that the support of an idempotent measure is contained in a compact subgroup. Conversely, it is simple to reduce Theorem 2 to the case where $G$ is compact. If $\mu$ is absolutely continuous then it clearly is a finite sum of characters multiplied by Haar measure. The difficulty in general lies in analyzing the singular part of $\mu$. Here the main point is to show that $\mu$ has mass on a closed subgroup of infinite index. In the case that $\hat{G}$ has no elements of finite order, this statement is equivalent to saying that the set $E$ intersects some cyclic subgroup of $\hat{G}$ in an infinite set. For arbitrary $\hat{G}$ it is proved by more complicated means. In either case one needs a technique which will yield some restriction on the nature of the set $E$. It is of course true that $E$ can be an arbitrary finite set. Hence we can only hope to derive statements about the set $E$ which allow for a finite number of exceptions. Nevertheless, our technique yields statements concerning finite sums of characters. These we state for the circle group.

THEOREM 3. For some $K$, whenever $c_{j}$ are such that $\left|c_{j}\right| \geqq 1$, and $n_{j}$ are arbitrary distinct integers, we have 


$$
\int_{0}^{2 \pi}\left|\sum_{j=1}^{N} c_{j} e^{i n j x}\right| d x>K\left(\frac{\log N}{\log \log N}\right)^{1 / 8} .
$$

It is a conjecture of Littlewood that the inequality holds with $K \log N$ on the right side. Previously, however, it was not even shown that the left side tended to infinity as a function of $N$. Indeed in the course of the proof of Theorem 2 we actually need this fact. The proof of Theorem 3 is completely independent of any abstract considerations. It is accomplished by exhibiting finite linear combinations of exponentials, $\phi_{k}$, such that $\left|\phi_{k}\right| \leqq 1$ and yet, if $\mu$ denotes the measure

$$
\sum c_{j} e^{i n j x} d x
$$

$\int \phi_{k} d \mu$ is large. We use some general lemmas concerning measures together with a combinatorial argument concerning the distribution of the integers $n_{j}$. In the case of idempotent measures, the same type of argument is used to show that the set $E$ has many finite sets $P$ such that for all $x$ in $E$, there is some $p$ in $P$ such that $x+p$ lies in $E$. This, however, does not suffice to characterize $E$ and further arguments are necessary. The details are too complicated to give here but will appear in forthcoming publications.

\section{REFERENCES}

1. Arne Beurling, and Henry Helson, Fourier-Stieltjes transforms with bounded powers, Math. Scand. vol. 1 (1953) pp. 120-126.

2. Henry Helson, Isomorphisms of Abelian group algebras, Ark. Mat. vol. 2 (1953) pp. $475-487$.

3. - Note on harmonic functions, Proc. Amer. Math. Soc. vol. 4 (1953) pp. 686-691.

4. Walter Rudin, Idempotent measures on abelian groups, to appear in Pacific J. Math.

5. - The automorphisms and endomorphisms of the group algebra of the unit circle, Acta Math. vol. 95 (1956) pp. 39-55.

6. - On isomorphisms of group algebras, Bull. Amer. Math. Soc. vol. 65 (1958) pp. 167-169.

7. R. Salem, On a problem of Littlewood, Amer. J. Math. vol. 7 (1955) pp. 535-540.

Massachusetts Institute of Technology 